\title{
Elongational viscosity of monodisperse and bidisperse polystyrene melts
}

\author{
Nielsen, Jens Kromann; Rasmussen, Henrik K.; Hassager, Ole; McKinley, G.H.
}

Published in:

Journal of Rheology

Link to article, DOI:

$10.1122 / 1.2206711$

Publication date:

2006

Document Version

Publisher's PDF, also known as Version of record

Link back to DTU Orbit

Citation (APA):

Nielsen, J. K., Rasmussen, H. K., Hassager, O., \& McKinley, G. H. (2006). Elongational viscosity of monodisperse and bidisperse polystyrene melts. Journal of Rheology, 50(4), 453-476.

https://doi.org/10.1122/1.2206711

\section{General rights}

Copyright and moral rights for the publications made accessible in the public portal are retained by the authors and/or other copyright owners and it is a condition of accessing publications that users recognise and abide by the legal requirements associated with these rights.

- Users may download and print one copy of any publication from the public portal for the purpose of private study or research.

- You may not further distribute the material or use it for any profit-making activity or commercial gain

- You may freely distribute the URL identifying the publication in the public portal

If you believe that this document breaches copyright please contact us providing details, and we will remove access to the work immediately and investigate your claim. 


\title{
Elongational viscosity of monodisperse and bidisperse polystyrene melts
}

\author{
Jens Kromann Nielsen \\ The Danish Polymer Centre, Department of Chemical Engineering, \\ Technical University of Denmark, DK-2800 Kgs. Lyngby, Denmark
}

Henrik Koblitz Rasmussen

The Danish Polymer Centre, Department of Manufacturing Engineering and Management, Technical University of Denmark, DK-2800 Kgs. Lyngby, Denmark

$$
\text { Ole Hassager }{ }^{a}
$$

The Danish Polymer Centre, Department of Chemical Engineering, Technical University of Denmark, DK-2800 Kgs. Lyngby, Denmark

\author{
Gareth H. McKinley
}

Hatsopoulos Microfluids Laboratory, Department of Mechanical Engineering, Massachusetts Institute of Technology, Cambridge, Massachusetts 02139

(Received 8 November 2005; final revision received 18 March 2006)

\section{Synopsis}

The start-up and steady uniaxial elongational viscosity have been measured for two monodisperse polystyrene melts with molecular weights of 52 and $103 \mathrm{~kg} / \mathrm{mole}$, and for three bidisperse polystyrene melts. The monodisperse melts show a maximum in the steady elongational viscosity vs. the elongational rate, $\dot{\epsilon}$, of about two times the limiting value of $3 \eta_{0}$ expected for a Newtonian fluid, whereas the bidisperse melts have a maximum of up to a factor of seven times the Trouton limit of $3 \eta_{0}$. The Wiest model which incorporates anisotropic drag and finite extensibility may be used to interpret the results in molecular terms. (c) 2006 The Society of Rheology.

[DOI: $10.1122 / 1.2206711]$

\section{INTRODUCTION}

The scaling of linear viscoelastic properties such as the zero shear viscosity, $\eta_{0}$, and the characteristic reptation time, $\tau_{d}$, for the Doi-Edwards model (Doi and Edwards, 1986) have been investigated thoroughly both theoretically and experimentally in the literature for monodisperse polymer melts. It is commonly accepted that the zero shear viscosity and the reptation time both scale with the molecular weight as $\eta_{0} \sim M^{3.4}$ and $\tau_{d} \sim M^{3.4}$ for monodisperse polymers with molecular weights substantially above the entanglement

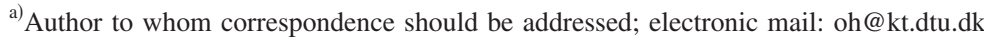


molecular weight, $M>(2-4) M_{e}$. Elongational flow properties have however not been analyzed as intensely. Thorough investigation of the elongational viscosity for very diluted solutions of monodisperse (and bidisperse) polystyrene have been made and analyzed by Gupta et al. (2000) and Ye et al. (2003). Wagner et al. (2005) have recently published elongational results for bidisperse blends of small amounts of ultra high, narrow molecular weight polystyrene, $M_{w}=3220 \mathrm{~kg} / \mathrm{mole}$ and $M_{w}=15400 \mathrm{~kg} / \mathrm{mole}$ dissolved in lower molecular weight polydisperse polystyrene, $M_{w}=423 \mathrm{~kg} / \mathrm{mole}$. Steady state was never reached, but the authors found that the blends were more strain hardening than the monodisperse melts, and that the maximum amount of strain hardening increased with increasing content of ultra high molecular weight polystyrene. To our knowledge the only published steady elongational viscosities for monodisperse melts are those of Bach et al. (2003a) and Luap et al. (2005). Neither the Doi-Edwards model nor other reptationbased models [Marrucci and Grizzutti (1988), Mead et al. (1998), Fang et al. (2000), Ianniruberto and Marrucci (2001), Schieber et al. (2003)] have effectively been able to predict the flow behavior of especially high Deborah-number flows, i.e., fast elongational flows with $\dot{\epsilon} \geq 1 / \tau_{d}$. Indeed, the major limitation to progress in the understanding of the nonlinear properties in elongational flow seems to be the scarcity of data for wellcharacterized narrow molecular weight linear polymer melts.

There have been a number of recent efforts at extending the basic reptation picture to incorporate additional physical mechanisms that modify the evolution in the polymeric stress in strong stretching flows. These include incorporating the role of "intrachain pressure" within a differential framework (Marrucci and Ianniruberto, 2004) and within the integral molecular stress function formulation (Wagner et al., 2005) or through detailed analysis of the rate of creation and destruction of "slip links" (Likhtman, 2005). The key change that each of these models seeks to incorporate is a modification in the scaling of the steady elongational steady stress with the elongational rate, $\sigma_{z z}-\sigma_{r r} \sim \dot{\epsilon}^{n}$. The bare reptation model of Doi and Edwards predicts a saturation in the stress, $n=0$ (corresponding to thinning in the elongational viscosity). Incorporation of chain stretching results in unbounded stress growth, which can be truncated through considering the finite extensibility of the chains resulting ultimately in $n=1$ (Fang et al., 2000) corresponding to finite limiting value of the elongational viscosity. The models proposed by Marrucci and Ianniruberto (2004) and Wagner et al. (2005) both find that $n=0.5$. In the present work we use the simple model proposed by Wiest (1989), which models the effects of the surroundings chains as an anisotropic drag acting on a finitely extensible dumbbell that represents a single segment of the orienting and elongating chain. This computationally simple model gives $n=0.5$, and we show below that it is able to capture many of the important features that we observe in the steady elongational viscosity.

Bach et al. (2003a) measured the elongational viscosity of two narrow molar mass distribution polystyrene melts, with $M_{w}=200 \mathrm{~kg} / \mathrm{mole}, \mathrm{PS} 200 \mathrm{~K}$, and $M_{w}=390 \mathrm{~kg} / \mathrm{mole}$, PS390K. The main conclusions drawn from this work were (1) The steady elongational viscosity for Deborah numbers, defined as $\mathrm{De}=\dot{\boldsymbol{\epsilon}} \tau_{d}$ greater than unity scales as $\bar{\eta}$ $\sim \dot{\epsilon}^{-0.5}$. (2) The steady elongational viscosity scales linearly with the molecular weight for $\mathrm{De}>1$, i.e., $\bar{\eta} \sim M_{w} \dot{\epsilon}^{-0.5}$, and finally (3) the steady elongational viscosity is a monotone decreasing function of the elongational rate. That is, $\bar{\eta}$ does not exceed $3 \eta_{0}$ for any elongational rate accessed experimentally.

The authors did point out that their conclusions with regard to molecular mass scaling were based on merely two samples. Based on the scaling properties of $\eta_{0}$ and $\tau_{d}$ with the molecular weight, it is however realized that these conclusions cannot be true if one extends them to elongational measurements of lower molecular weights. There are simply 
TABLE I. Composition of blend 1, blend 2, blend 3, Ye et al.'s blend, and Wagner et al.'s blends.

\begin{tabular}{lcccccc}
\hline \hline & Blend 1 & Blend 2 & Blend 3 & Ye et al. & Wagner I & Wagner II \\
\hline w/w\% PS50K & 95.98 & 85.63 & 0 & -- & - & - \\
w/w\% PS100K & 0 & 0 & 85.98 & - & - & - \\
w/w\% PS390K & 4.02 & 14.37 & 14.02 & -- & - & - \\
$c_{P S 390 K} / \mathrm{c}^{*}$ & 2.5 & 10 & 10 & $-\ldots$ & - & - \\
$M_{w}[\mathrm{~kg} / \mathrm{mol}]$ & 65.3 & 100.3 & 143.1 & - & - & - \\
$G_{r}$ & 0.499 & 0.499 & 0.064 & 0.0192 & 0.0075 & 0.0357 \\
\hline \hline
\end{tabular}

too many constraints. Marrucci et al. (2004) have treated this problem theoretically and suggested that melts with fewer entanglements may show a maximum in $\bar{\eta}$ as function of $\dot{\epsilon}$.

The first purpose of this work is to investigate how two polystyrene melts with $M_{w}=103 \mathrm{~kg} / \mathrm{mole}, \mathrm{PS} 100 \mathrm{~K}$, and $M_{w}=52 \mathrm{~kg} / \mathrm{mole}$, PS50K, behave in a uniaxial elongational flow at $130{ }^{\circ} \mathrm{C}$. Polystyrene has an entanglement molecular weight of $M_{e}=13.3 \mathrm{~kg} / \mathrm{mole}$ (Fetters et al., 1994), giving the melts respectively, 7.7 and 3.9 entanglements. With these fluids it is possible to analyze what happens to $\bar{\eta}$ in the transition going from low to high Deborah numbers, i.e., from the linear to the nonlinearly dominated regime. The elongational measurements can give an indication of which of the constraints noted above must be relaxed.

The reptation time for PS100K is $\tau_{d} \approx 100 \mathrm{~s}$ at $130{ }^{\circ} \mathrm{C}$. The range over which the elongational rates can be measured by the filament stretching rheometer (FSR) to avoid dissipative heating in the sample is $\dot{\epsilon} \leq 0.3 \mathrm{~s}^{-1}$ (Bach et al., 2003a), making it possible to transition from low to high Deborah numbers for PS100K. The lower molecular weight PS50K sample is expected mostly to provide information about the linear region, since $\tau_{d} \approx 10 \mathrm{~s}$.

The lack of a maximum in $\bar{\eta}$ vs $\dot{\epsilon}$ for PS200K and PS390K is believed to be related to the monodisperse character of the melts, and it has therefore been decided to make three bidisperse melts, in which each of the individual polymers in the blend is expected not to display a maximum in $\bar{\eta}$ vs $\dot{\epsilon}$ when studied in isolation. We have decided to mix PS390K with PS50K in two different concentrations in order to investigate the effect of diluting PS390K with PS50K. Second, we have made a mixture of PS390K with PS100K, where PS390K has the same mass concentration as one of the PS390K+PS50K blends.

The compositions of the three blends used in this work are shown in Table I. In this table we also show the concentration of PS390K relative to the overlap concentration, $c^{*}$, of PS390K in a dilute solution under theta conditions defined by Doi (1992) (page 20). The radius of gyration $R_{g}$ for PS390K is found to be $R_{g}=168 \AA$ (Fetters et al., 1994), and since one polymer has a volume of order $O\left[\left(2 R_{g}\right)^{3}\right]$, the overlap concentration of PS390K is found to be $c^{*}=16 \mathrm{~kg} / \mathrm{m}^{3}$, or $c^{*}=1.6 \mathrm{w} / \mathrm{w} \%$. We also specify in Table I the weightaverage molecular weight of the bidisperse blends, $M_{w}=\phi_{L} M_{L}+\phi_{S} M_{S}$, where $\phi_{i}$ and $M_{i}$ are the weight fractions and the molecular weights of the long-chain $(L)$ and short-chain $(S)$ components.

\section{EXPERIMENT}

\section{A. Synthesis and chromatography}

The two polystyrene samples PS50K and PS100K were synthesized by anionic polymerization (Ndoni et al., 1995). The molecular weights were determined by size exclu- 
TABLE II. Molecular weights $\left(M_{w}\right)$ and polydispersities $\left(M_{w} / M_{n}\right)$ of the pure and blended polystyrene melts.

\begin{tabular}{lccccccc}
\hline \hline \multicolumn{1}{c}{ Name } & PS50K & PS100K & PS200K & PS390K & Blend 1 & Blend 2 & Blend 3 \\
\hline$M_{w}[\mathrm{~kg} / \mathrm{mol}]$ & 51.7 & 102.8 & 200.0 & 390.0 & 65.3 & 100.3 & 143.1 \\
$M_{w} / M_{n}$ & 1.026 & 1.022 & 1.040 & 1.060 & 1.218 & 1.683 & 1.248 \\
\hline \hline
\end{tabular}

sion chromatography (SEC) with toluene as the eluent using a Viscotec 200 instrument equipped with a PLguard and two PLgel mixed D columns in series (from Polymer Laboratories) using a RI detector. On the basis of calibration with narrow molecular weight polystyrene standards, the values of $M_{w}$ and $M_{w} / M_{n}$ were measured for the monodisperse samples. The results are given in Table II.

\section{B. Mechanical spectroscopy}

The viscoelastic properties of the polystyrene melts were obtained from smallamplitude oscillatory shear flow measurements on an AR2000 rheometer from TA instruments using a plate-plate geometry (see Figs. 1 and 2). The measurements were performed at $130{ }^{\circ} \mathrm{C}$ for the PS50K, PS100K, and blends, and at $150{ }^{\circ} \mathrm{C}$ for the blends. The measured data at $150{ }^{\circ} \mathrm{C}$ were shifted to $130{ }^{\circ} \mathrm{C}$ using the time-temperature superposition shift factor $a_{T}$ found from the WLF equation (Bach et al., 2003a);

$$
\log _{10}\left(a_{T}\right)=\frac{-c_{1}^{0}\left(T-T_{0}\right)}{c_{2}^{0}+\left(T-T_{0}\right)},
$$

where $c_{1}^{0}=8.86, c_{2}^{0}=101.6^{\circ} \mathrm{C}, T_{0}=136.5^{\circ} \mathrm{C}$, and $T$ is the sample temperature in ${ }^{\circ} \mathrm{C}$.

\section{Transient elongational viscosity measurements}

The transient elongational viscosity was measured using a filament stretching rheometer which is described in detail elsewhere (Bach et al., 2003b). The polystyrene melts were dried according to the protocol of Schausberger and Schindlauer (1985), and molded into cylindrical-shaped samples, with radius of $R_{i}=4.5 \mathrm{~mm}$ and height of $L_{i}$

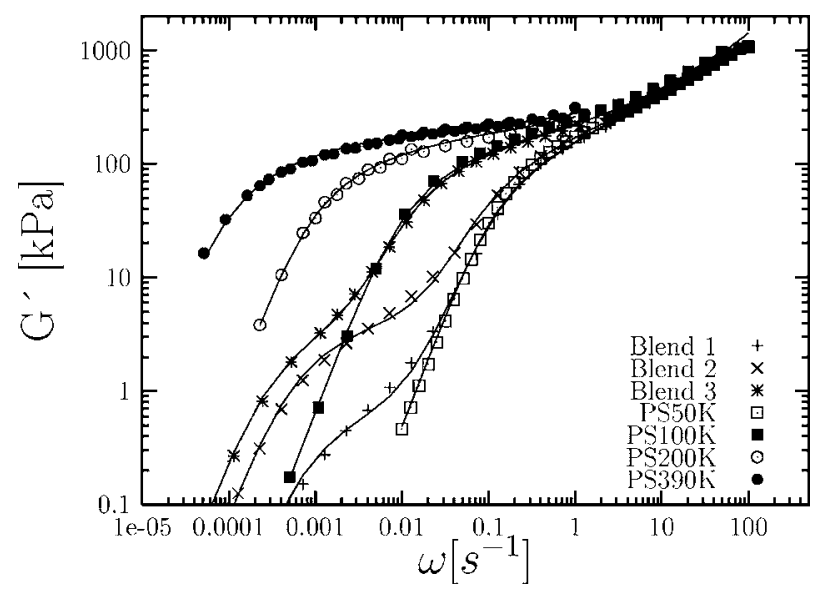

FIG. 1. Results of linear viscoelastic measurements of $G^{\prime}$ as a function of the angular frequency $\omega$. The measurements on the polystyrene melts were performed at 130,150 , and $170{ }^{\circ} \mathrm{C}$. The data are all timetemperature shifted to a reference temperature of $T_{0}=130^{\circ} \mathrm{C}$. 


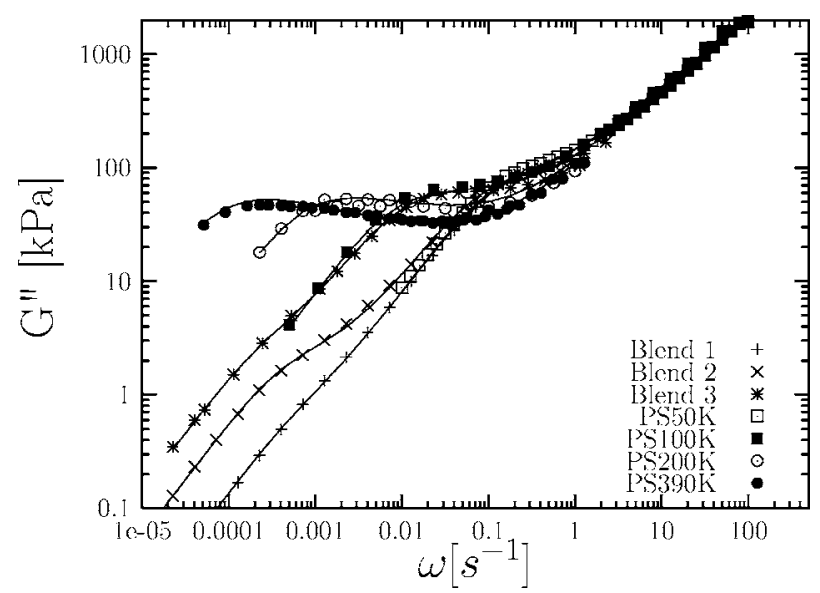

FIG. 2. Results of linear viscoelastic measurements of $G^{\prime \prime}$ as a function of the angular frequency $\omega$. The measurements on the polystyrene melts were performed at 130,150 , and $170{ }^{\circ} \mathrm{C}$. The data are all timetemperature shifted to a reference temperature of $T_{0}=130{ }^{\circ} \mathrm{C}$.

$=2.5 \mathrm{~mm}$ using a Carver hydraulic press. The PS50K and PS100K samples were pressed at $150{ }^{\circ} \mathrm{C}$ and annealed at this temperature for $2 \mathrm{~min}$. The bidisperse blends were pressed and annealed for $2 \mathrm{~min}$ at $170^{\circ} \mathrm{C}$. The temperatures were chosen to ensure that the polymer chains were completely relaxed but did not degrade; this was confirmed using SEC after the elongational experiment was performed. The molded pellets were placed between two parallel plates inside the filament stretching rheometer, and the temperature was raised to $130{ }^{\circ} \mathrm{C}$. To ensure adhesion between the end plates and polymer melt, the end plates were coated with a solution of polystyrene in tetrahydrofuran as described in Bach et al., (2003a). In most of the experiments performed, the sample was prestretched in order to reduce the transmitted force in the vertical plane to avoid the sample being ripped off the end plates. All samples were prestretched by variable amounts, thus the initial radius for experiments with PS100K at $\dot{\epsilon}=0.3 \mathrm{~s}^{-1}$ was $R_{0}=1.5 \mathrm{~mm}$, whereas the initial radius for Blend 3 was $R_{0}=4.3 \mathrm{~mm}$ at $\dot{\epsilon}=0.00015 \mathrm{~s}^{-1}$. The prestretch was performed with stretch rates considerably lower than the inverse of the longest relaxational time. The melt is allowed to relax before every elongational experiment is started. We wait until all residual orientation in the polymer has disappeared, which is the case when no residual forces are present as indicated by the load cell. This equilibration time is at least ten times the longest relaxation time of the melt.

During a stretching experiment a laser micrometer samples the central diameter of the elongating filament while a load cell measures the force at the end plate. The diameter data are sent directly to a controller that produces a signal to the motor pulling the end plates apart. This control method ensures that the radius decreases exponentially with time as $R(t)=R_{0} e^{-\dot{\epsilon} t / 2}$. The Hencky strain is defined as $\epsilon=-2 \ln \left[R(t) / R_{0}\right]$. After an elongational experiment is complete, the measured radius $R(t)$ and force $F(t)$ are used to calculate the tensile stress,

$$
\sigma_{z z}-\sigma_{r r}=\frac{F(t)-m_{1} g}{\pi R(t)^{2}}
$$

and the transient elongational viscosity as 


$$
\bar{\eta}^{+}(t)=\frac{\sigma_{z z}-\sigma_{r r}}{\dot{\epsilon}},
$$

where the measured force, $F$, is corrected by the weight of the lower half of the polymer filament, $m_{1}$ and the gravitational acceleration $g$ (Szabo, 1997). This weight is measured by forcing the filament to break at the symmetry plane after the end of an experiment.

At small strains there is an extra force contribution from the shear components in the deformation field during start-up. The shear component originates from the no-slip condition at the rigid end plates and is especially important at small aspect ratios. For Newtonian fluids this reverse squeeze-flow problem can be modeled analytically and the effect of the additional shear may be eliminated by a correction factor [Spiegelberg and McKinley (1996)].

$$
\bar{\eta}_{\mathrm{corr}}^{+}=\bar{\eta}^{+}\left(1+\frac{\exp \left(-7\left(\epsilon+\epsilon_{0}\right) / 3\right)}{3 \Lambda_{i}^{2}}\right)^{-1},
$$

where $\Lambda_{i}=L_{i} / R_{i}$ is the initial aspect ratio, $\epsilon_{0}$ is the prestretched Hencky strain, defined as $\epsilon_{0}=-2 \ln \left(R_{0} / R_{i}\right)$ and $\bar{\eta}_{\text {corr }}^{+}$is the corrected transient uniaxial elongation viscosity.

This correction is analytically correct for very small strains $(\epsilon \rightarrow 0)$ for all types of fluids. However, the correction is less accurate at increasing strains where the effect of the correction fortunately vanishes.

In this work we have chosen to present the elongational measurements in both uncorrected and corrected form, as we also prefer to present the raw data. For the aspect ratio used here, the extra force contribution is negligible after about one additional strain unit. This was demonstrated experimentally in Bach et al. (2003b), and theoretically in Kolte et al. (1997) for polymer melts.

Eriksson and Rasmussen (2005) suggest that the relevant nondimensional measure of the surface tension in viscoelastic flow is the ratio of the surface tension stresses to the complex modulus $G^{*}(\omega)=\sqrt{G^{\prime}(\omega)^{2}+G^{\prime \prime}(\omega)^{2}}$, i.e., $V_{C}=\sigma /\left[R G^{*}(\dot{\epsilon})\right]$, where the angular frequency, $\omega$, has been replaced with the characteristic deformation rate, $\dot{\epsilon}$. This viscoelastic capillary number resembles the surface elasticity number [Spiegelberg and McKinley (1996) and Rasmussen and Hassager (2001)] at high deformation rates and the inverse of the classical capillary number at low deformation rates. As $V_{C}$ stays below 0.03 in all experiments, the effect of surface tension is negligible.

The effect of gravitational sagging can be evaluated using a relevant measure of the magnitude of gravitational forces relative to the viscous forces. Here, we use the ratio $L_{i} \exp \left(\epsilon+\epsilon_{0}\right) \rho g /\left(2 \dot{\epsilon} \bar{\eta}^{+}\right)$as in Rasmussen et al. (2005), where $\rho$ is the density of the polymer melt. The duration of the elongational experiments in this work was considerably below the sagging time, as this number is less than 0.1 in all the performed experiments. See Szabo and McKinley (2003), for additional discussion of similar correction factors.

\section{LINEAR VISCOELASTIC MEASUREMENTS}

A linear viscoelastic (LVE) analysis provides us with an estimate of the elongational behavior in the limit De $\rightarrow 0$ and provides a verification of the reliability of the elongational experiments especially at short times and small strains. If the verifications of the experiments were the sole purpose of doing LVE experiments a simple Maxwell fit to the data would be sufficient. But, we also seek to determine the characteristic time constants of the individual polymeric species in the melt, and for this the Baumgaertel, Schausberger, and Winter (BSW) model is used (Baumgaertel et al., 1990). Each polymer 
TABLE III. Linear viscoelastic properties of the pure and blended melts at $130{ }^{\circ} \mathrm{C}$. The constants in the BSW model are $n_{e}=0.23, n_{g}=0.67$, and $\lambda_{c}=0.4 \mathrm{~s}$ as obtained from Jackson and Winter (1995) plus $G_{N}^{0}=250 \mathrm{kPa}$.

\begin{tabular}{lccccccc}
\hline \hline \multicolumn{1}{c}{ Name } & PS50K & PS100K & PS200K & PS390K & Blend 1 & Blend 2 & Blend 3 \\
\hline$\eta_{0,1}[\mathrm{MPa}]$ & 0.82 & 7.88 & 82.9 & 724 & 0.78 & 1.02 & 5.97 \\
$\eta_{0,2}[\mathrm{MPa}]$ & -- & --- & --- & --- & 0.59 & 4.64 & 8.58 \\
$\eta_{0}[\mathrm{MPa}]$ & 0.82 & 7.88 & 82.9 & 724 & 1.37 & 5.66 & 14.6 \\
$\lambda_{\max , 1}[\mathrm{~s}]$ & 12.8 & 158 & 1749 & 15441 & 12.2 & 17.4 & 122.1 \\
$\lambda_{\max , 2}[\mathrm{~s}]$ & & & & & 2186 & 3182 & 5572 \\
$\lambda_{a, 1}[\mathrm{~s}]$ & 7.05 & 87.02 & 965 & 8517 & 6.73 & 9.60 & 67.4 \\
$\lambda_{a, 2}[\mathrm{~s}]$ & -- & -- & --- & --- & 1206 & 1755 & 3074 \\
$G_{N 1}^{0}[\mathrm{kPa}]$ & 250 & 250 & 250 & 250 & 249 & 242 & 242 \\
$G_{N 2}^{0}[\mathrm{kPa}]$ & -- & --- & --- & --- & 1.43 & 7.73 & 8.18 \\
$G_{N}^{0}[\mathrm{kPa}]$ & 250 & 250 & 250 & 250 & 250 & 250 & 250 \\
\hline \hline
\end{tabular}

contributes a distinct spectrum with a characteristic time constant. We analyze the LVE data with a theoretical approach suggested by Jackson and Winter (1995) which handles mono- and bidisperse melts. This is not to be confused with a blend rule, since the LVE properties of the blends cannot be predicted from the composition of long- and short polymers by this procedure. The LVE properties of monodisperse linear polymers (Milner and McLeish, 1998) and mixing rules for blends of monodisperse species [des Cloizeaux (1988)] have been studied in detail. In terms of physical insight the BSW approach is not far from a simple Maxwell fit, with a few exceptions as described later.

The relaxation modulus $G(t)$ is found from the continuous-spectrum $H(\lambda)$, which for the bidisperse blends is composed of two individual spectra,

$$
\begin{gathered}
G(t)=G_{1}(t)+G_{2}(t), \\
G_{i}(t)=\int_{0}^{\infty} \frac{H_{i}(\lambda)}{\lambda} \exp (-t / \lambda) d \lambda, \quad i=1,2, \\
H_{i}(\lambda)=n_{e} G_{N, i}^{0}\left[\left(\frac{\lambda}{\lambda_{\max , i}}\right)^{n_{e}}+\left(\frac{\lambda}{\lambda_{c}}\right)^{-n_{g}}\right] h\left(1-\lambda / \lambda_{\max , i}\right) .
\end{gathered}
$$

Here, $h(x)$ is the Heaviside step function, $n_{e}$ is the slope of the $\left[\log (\omega), \log G^{\prime}\right]$ curve at intermediate frequencies $\omega, n_{g}$ is the slope of $\left[\log (\omega), \log G^{\prime \prime}\right]$ for $\omega \rightarrow \infty$, and $\lambda_{c}$ is called the crossover relaxation time. We constrain the individual contributions to the modulus in a way such that $G_{N}^{0}=G_{N, 1}^{0}+G_{N, 2}^{0}$ is constant.

When least-squares fitting (Rasmussen et al., 2000) the BSW model to the LVE data, $n_{e}, n_{g}$ (both independent of temperature), $\lambda_{c}$, and $G_{N}^{0}$ are treated as fixed values. The crossover time $\lambda_{c}$ depends on temperature in the same way as any other relaxation time. We use $n_{e}=0.23, n_{g}=0.67$ and $\lambda_{c}=0.4 \mathrm{~s}$ (at $130{ }^{\circ} \mathrm{C}$ ) as obtained by Jackson and Winter (1995). The value of $G_{N}^{0}$ was found by Bach et al. (2003a) to be $250 \mathrm{kPa}$ at $130{ }^{\circ} \mathrm{C}$, and we have decided to use this value as a fixed parameter. This means that the only remaining adjustable parameters to model the LVE data are the two largest relaxation times, $\lambda_{\max , 1}$ and $\lambda_{\max , 2}$ as seen in Table III.

Since the monodisperse melts only have one largest time constant $\lambda_{\max }$, this is the single adjustable parameter for fitting the LVE data for PS50K, PS100K, and PS390K to the BSW model. In order to be able to compare the properties of monodisperse and bidisperse melts, the same values of $n_{e}, n_{g}$, and $\lambda_{c}$ are always used. The model parameters 
are given in Table III, obtained by least-squares fitting the measured values of $G^{\prime}$ and $G^{\prime \prime}$. The experimental results for $G^{\prime}$ and $G^{\prime \prime}$ are shown in Figs. 1 and 2 together with the best fit of the BSW model. The zero shear viscosities are calculated as

$$
\eta_{0, i}=\int_{0}^{\infty} G_{i}(s) d s=n_{e} G_{N, i}^{0} \lambda_{\max , i}\left(\frac{1}{1+n_{e}}+\frac{1}{1-n_{g}}\left(\frac{\lambda_{\max , i}}{\lambda_{c}}\right)^{-n_{g}}\right) .
$$

For the monodisperse melts $i=1$. For the bidisperse melts $i=1,2$, and the individual $\eta_{0, i}$ can be added to find the actual, measured value of $\eta_{0}=\eta_{0,1}+\eta_{0,2}$.

Fitting $\eta_{0}$ for the monodisperse melts PS50K, PS100K, PS200K, and PS390K with the molecular weight as a power law, the exponent is found to be 3.38 as generally observed for these moderately entangled systems.

The average reptation time is calculated as:

$$
\lambda_{a, i}=\frac{\int_{0}^{\infty} G_{i}(s) s d s}{\int_{0}^{\infty} G_{i}(s) d s} \approx \lambda_{\max , i}\left(\frac{1+n_{e}}{2+n_{e}}\right) .
$$

This expression applied to the Doi-Edwards relaxation modulus gives a value that is within $2 \%$ of the commonly denoted reptation time, $\tau_{d}$. This time is found to scale with molecular weight as $\lambda_{a} \sim M^{3.52}$ for our monodisperse melts.

The characteristic time constants for the bidisperse systems found in Table III show that the smaller time constant in the blend is more or less unchanged compared to the time constant for the undiluted small molecular weight melt. This is in agreement with the expectation (Doi et al., 1987) that there will be no tube dilation for the short chains. By contrast, the longest relaxation time in the blend has been significantly reduced compared to the longest relaxation time for an undiluted melt of long chains which is attributed to the effect of tube dilation, (Doi et al. 1987).

Struglinksi and Graessley (1985) have predicted that when the molar masses in a binary blend of short $\left(M_{s}\right)$ and long $\left(M_{l}\right)$ chains are far apart, the reptation time for the longest molecules should not depend on the blend composition. The relevant constraint release parameter is defined as $G_{r}=M_{L} M_{e}^{2} / M_{S}^{3}$, with the prediction that the reptation time of the longer chains should be unchanged provided $G_{r}<0.1$. More recent investigations (Lee et al., 2005 and Park and Larson, 2004), however, suggest that the critical condition is somewhat lower with $G_{r c} \approx 0.064$ such that the nondilation regime is limited to $G_{r}$ $<G_{r c}$. The constraint release parameters for our blends (shown in Table I) are indeed all larger than $G_{r c}$ indicating that tube dilation takes place and that relaxation of the stress carried by the long chains is the result of constraint release due to reptation of the short chains. This is reflected in the values of $\lambda_{a, 2}$ for the blends compared to the value $\lambda_{a, 1}$ for the pure long chains (PS390K) in Table III. By contrast, the short relaxation time of blend $\left(\lambda_{a, 1}\right)$ is substantially unchanged compared to that of the pure short chains, indicating that the short chains are reptating in an essentially frozen network of long chains. Moreover, according to the revised Struglinski and Graessley criterion our blend 3 should be the least affected by tube dilation, also in agreement with observations.

Struglinski and Graessley also conclude that the zero shear viscosity $\eta_{0}$ for bidisperse melts depends on the weight-average molecular weights of the monodisperse melts where $\eta_{0} \sim M_{w}^{3.4}$. This prediction deviates less than $40 \%$ from our measured zero shear viscosities. 
Ye et al. (2003) used two monodisperse polystyrene samples of molar masses $M_{s}=2890 \mathrm{~kg} / \mathrm{mole}$ and $M_{l}=8420 \mathrm{~kg} /$ mole to prepare a series of bidisperse solutions spanning the range from pure short chains to long chains. All blends were dissolved in tricresyl phosphate with an overall polymer volume fraction of $7 \%$. These blends, all in the semidilute regime, were subsequently characterized in uniaxial extensional flow using a filament stretching device and successfully compared to the predictions of a simplified reptation model designed to investigate the effects of polydispersity. A characteristic feature of the steady elongational viscosity measured for all investigated solutions, including the monodisperse ones, was a transition to strain hardening, which was interpreted as a signature of chain stretching. In other words there was no qualitative difference between the measured elongational viscosity of the entangled monodisperse and bidisperse polystyrene solutions. The results obtained by Ye et al. (2003) are thus expected to differ from our study for at least two reasons. First, in Table I we show the values of the Struglinski-Graessley parameter $G_{r}$ for the blends studied by Ye et al. (2003). The widely disparate values of the reptation times for the two species lead to $G_{r c} \ll 1$ and indicate that, in contrast to our experiments, the dynamics of the longer chain should remain unchanged regardless of the presence of the shorter species. Second, the materials studied by Ye et al. (2003) are semidilute entangled solutions rather than melts. Even though the number of entanglements is comparable, the higher molecular weight of each entangled segment when diluted by a solvent results in a greater number of Kuhn steps in each segment and consequently a larger molecular extensibility (Appendix Sec. 2). For completeness, we have also included the Graessley parameters for Wagner et al.'s (2005) bidisperse melts in Table I.

\section{ELONGATIONAL VISCOSITY MEASUREMENTS}

\section{A. Startup of elongational flow}

Figures 3-5 show the corrected transient elongational viscosity [Eq. (4)] for PS50K, PS100K, blend 1, and blend 3 together with the LVE predictions, all measured at $130{ }^{\circ} \mathrm{C}$. The elongational measurements for all melts show good agreement with the LVE prediction at small strains. The deviation between the transient elongational data and LVE calculations is less than $15 \%$ in all measurements. Figures 6-9 show the same measurements as in Figs. 3-5, with uncorrected tensile stress differences [Eq. (3)] plotted against strain. It is seen that the steady elongational viscosity is obtained for all elongational rates. As the elongational rate increases, the plateau region is maintained for fewer strain units as compared to smaller rates. This is due to a larger prestretch, $\epsilon_{0}$, for the highstretch-rate experiments. The reason for increasing the prestretch in the faster experiments is twofold. First, it minimizes the magnitude of the correction for reverse squeeze flow at high rates [see Eq. (4)]. Second, it helps reduce the tendency for de-adhesion of the sample from the endplate. The adhesive force holding the sample to the end plate has a maximum value; by prestreching the sample to induce a neck at the midplane, higher tensile stresses (and hence higher maximum stretching rates) can then be tolerated in the middle of the filament for a given force of adhesion at the end plates.

\section{B. Steady viscosity scaling at intermediate Deborah numbers 1. Monodisperse melts}

We first turn our attention to the results for the monodisperse melts in order to compare with the results from Bach et al. (2003a). We see from Figs. 3 and 10 that the steady viscosity for PS100K reaches a value very close to $3 \eta_{0}$ for the lowest elongational rate. 


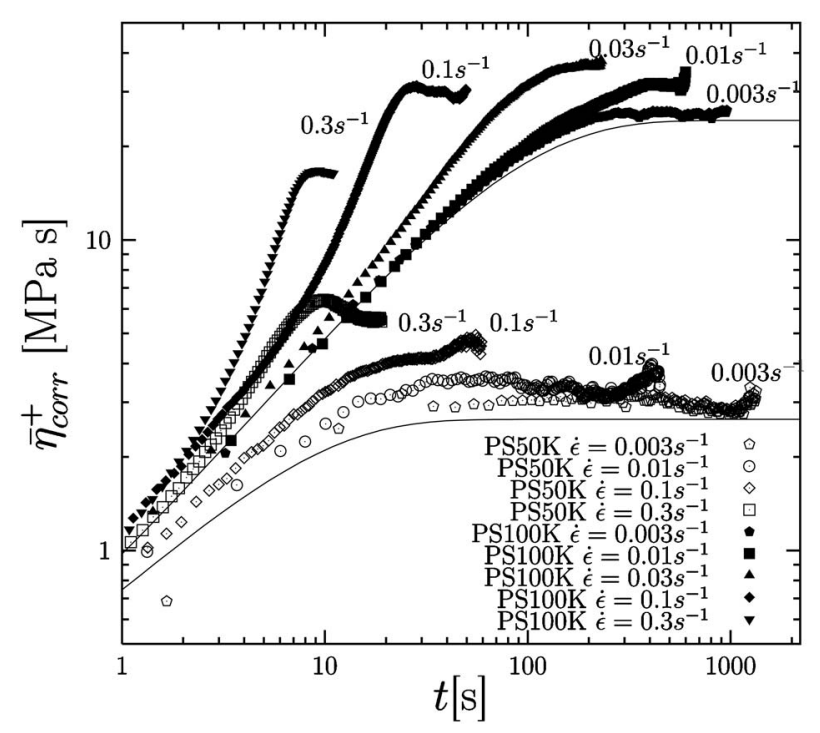

FIG. 3. Corrected [Eq. (4)] transient extensional viscosity of PS50K and PS100K measured at different strain rates. Measurements were performed at $130{ }^{\circ} \mathrm{C}$.

The time-dependent transient viscosity, $\bar{\eta}^{+}$, for the lowest rate follows the LVE prediction. At intermediate Deborah numbers, i.e. $1<\mathrm{De}<10$, the steady elongational viscosity $\bar{\eta}$ rises above $3 \eta_{0}$. For PS100K $\bar{\eta}$ is about $50 \%$ above $3 \eta_{0}$, and the $\bar{\eta}$-maximum is stretched over two decades of $\dot{\epsilon}$. The maximum for PS50K is measured to be at least $100 \%$ above $3 \eta_{0}$. It is possibly higher than $6 \eta_{0}$, since the highest measured elongational rate also gives the highest $\bar{\eta}^{+}$-value. The elongation rate at which the maximum occurs corresponds to a Deborah number around De $\approx 3$ for both melts (assuming that $\bar{\eta}$ reaches its maximum at a elongational rate somewhat higher than $\dot{\epsilon}=0.3 \mathrm{~s}^{-1}$ for PS50K).

The elongational viscosity measurements for PS50K are limited to De $<3$ because of the restriction due to dissipitative heating which limits the measurements to $\dot{\epsilon} \leq 0.3 \mathrm{~s}^{-1}$.

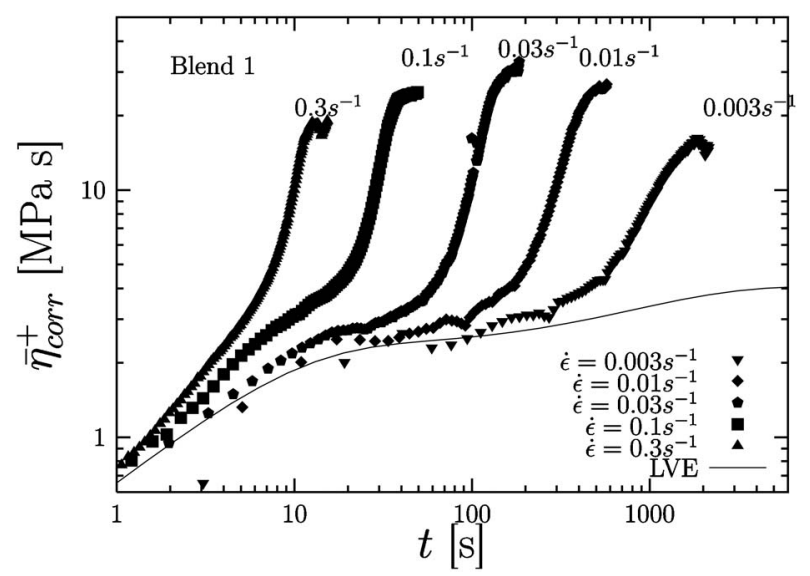

FIG. 4. Corrected transient extensional viscosity [Eq. (4)] of Blend 1 measured at different strain rates. Measurements were performed at $130{ }^{\circ} \mathrm{C}$. 


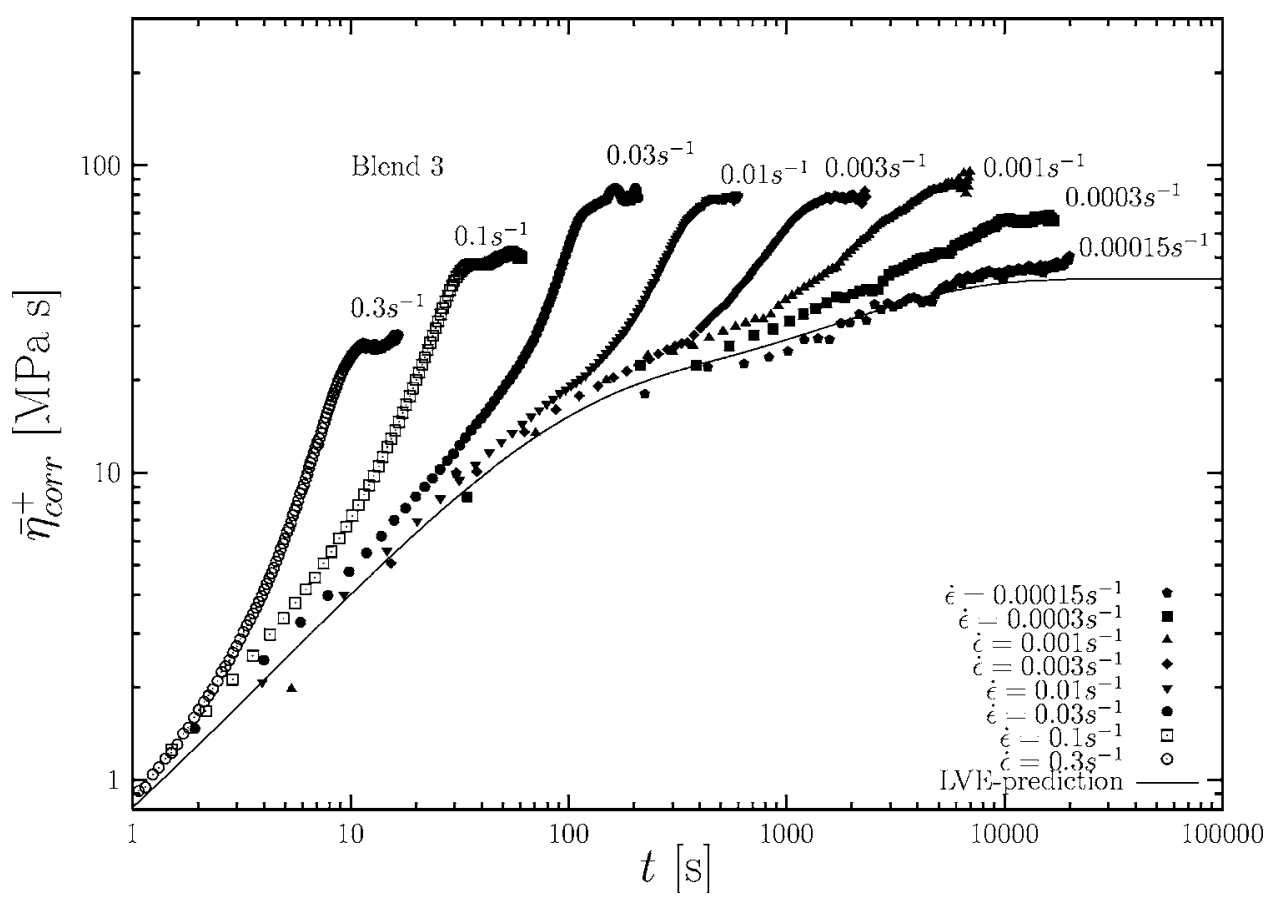

FIG. 5. Corrected transient extensional viscosity [Eq. (4)] of blend 3 measured at different strain rates. Measurements were performed at $130{ }^{\circ} \mathrm{C}$.

There are only two measurements in the nonlinear regime available for PS100K, and none for PS50K. This makes it difficult to compare with the scaling behavior proposed by Bach et al. (2003a).

Bach et al. (2003a) claimed that the steady elongational stress scaled linearly with the molecular weight at high Deborah numbers. This scaling can be illustrated by interpretating data according to the recently published theory of Marrucci and Ianniruberto (2004). Figure 11 shows steady values of $\left(\sigma_{z z}-\sigma_{r r}\right) / G_{N}^{0}$ vs. $\dot{\epsilon} \tau_{p}$ for all of the monodis-

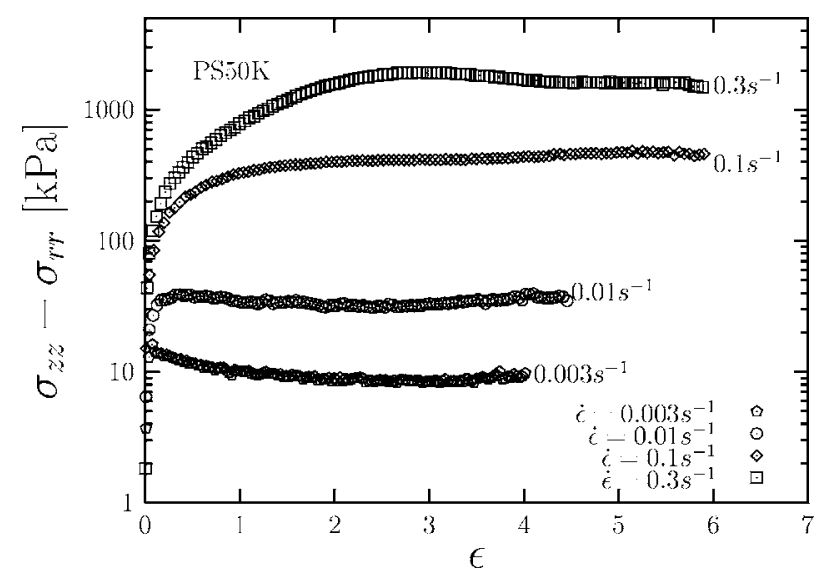

FIG. 6. Same data as in Fig. 3 for PS50K but plotted as uncorrected transient extensional stress [Eq. (3)] against Hencky strain $\epsilon$. 


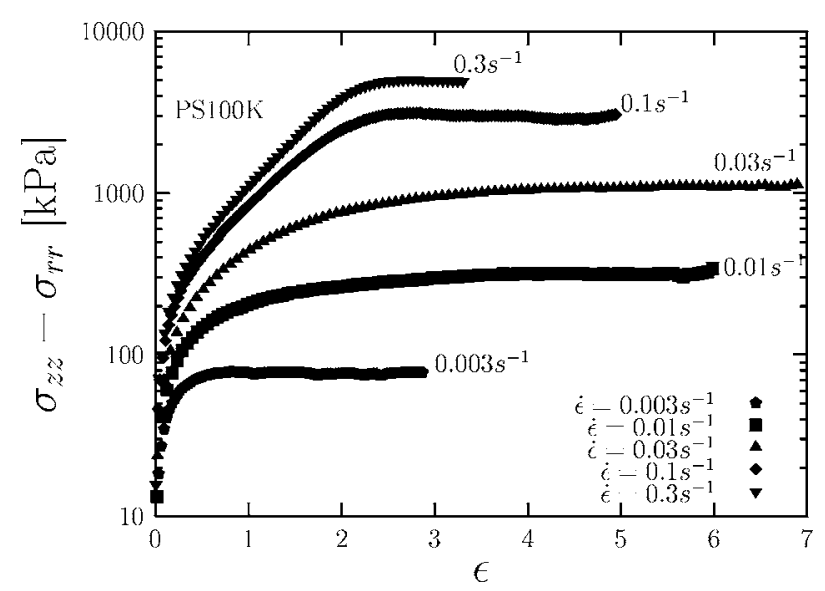

FIG. 7. Same data as in Fig. 3 for PS100K but plotted as uncorrected transient extensional stress [Eq. (3)] against Hencky strain $\epsilon$.

perse melts. Here, $\tau_{p}$ represents the relaxation time of the squeezing pressure effect as defined by Marrucci and Ianniruberto (2004). Marrucci and Ianniruberto report $\tau_{p}$ for PS200 to be $\tau_{p}=1000 \mathrm{~s}$, and the scaling is $\tau_{p} \sim M_{w}^{2}$. This is used to calculate $\tau_{p}$ for the other monodisperse melts, which then become: $\tau_{p}=66.8 \mathrm{~s}$ for PS50K, $\tau_{p}=264.2 \mathrm{~s}$ for PS100K, and $\tau_{p}=3802.5 \mathrm{~s}$ for PS390K. It is seen in Fig. 11 that the scaled values for PS50K and PS100K lie on the same line as the data for PS200K and PS390K steady-state stresses, hereby showing that the linear scaling of steady stress with molecular weight at high Deborah numbers is valid.

Another conclusion from the work of Bach et al. (2003a) was that the steady elongational viscosity scales approximately $\dot{\epsilon}^{-0.5}$ for large Deborah numbers. By examining the raw data from Bach et al. (2003a) more closely and performing a linear regression, it is however concluded that the exponent is $-0.42 \pm 0.03$ within a $95 \%$ confidence interval. In the present study there are only two measurements of $\bar{\eta}$ for PS100K that could confirm

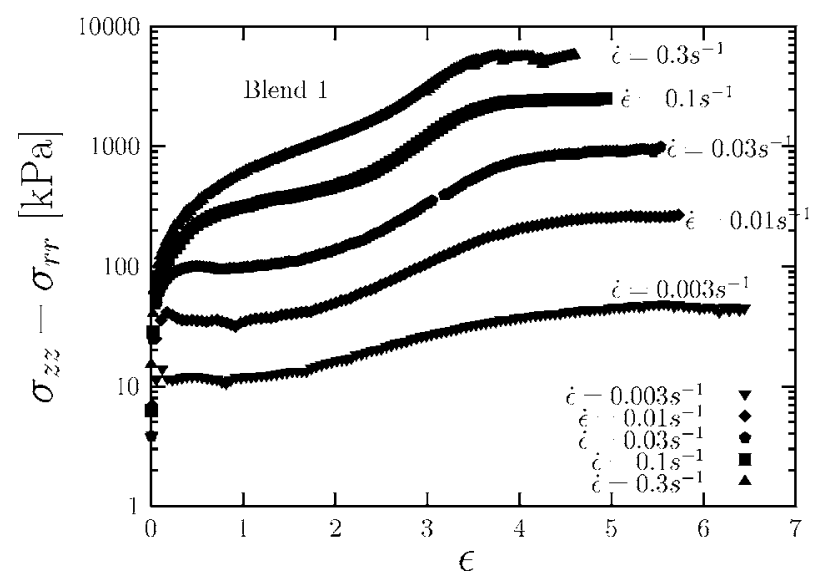

FIG. 8. Same data as in Fig. 4 for blend 1 but plotted as uncorrected transient extensional stress [Eq. (3)] against Hencky strain $\epsilon$. 


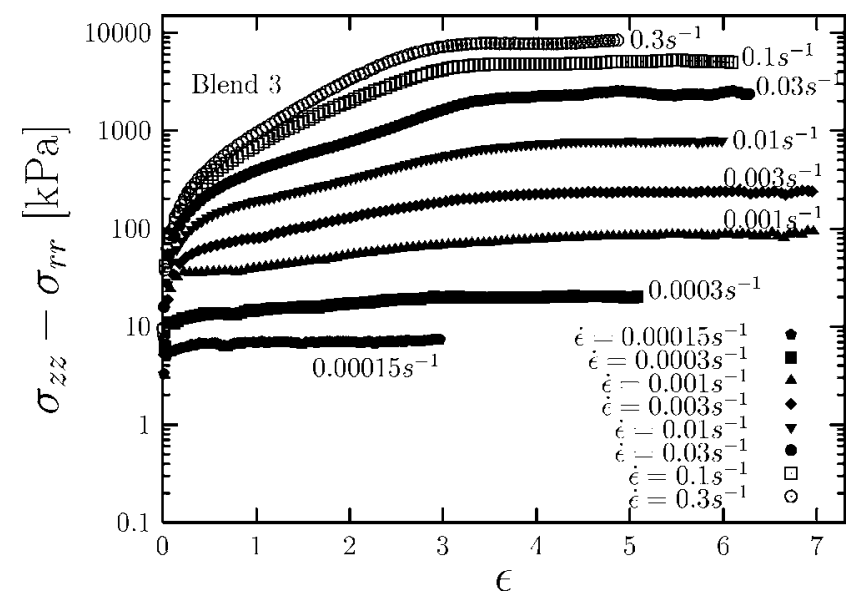

FIG. 9. Same data as in Fig. 5 for blend 3 but plotted as the uncorrected transient extensional stress [Eq. (3)] against Hencky strain $\epsilon$.

this power law behavior, and none for PS50K. Figure 10 shows the steady elongational viscosity of PS100K, and it appears to show the expected asymptotic behavior.

\section{Bidisperse melts}

Figure 4 and 5 show the corrected transient elongational viscosity for the blends denoted blend 1 and blend 3; see Table I. It can be seen from both plots that there is good agreement between the elongational measurements and the LVE prediction for small strains. The steady viscosity lies substantially above $3 \eta_{0}$ for all measurements, except for blend 3 at $\dot{\epsilon}=0.3 \mathrm{~s}^{-1}$.

The complex interdependence of the transient extensional rheology for the entangled blends on stretching rate, molecular weight and concentration is illustrated in Fig. 12 for the PS50K/PS390K blends. For a pure PS50K melt at a strain rate of $\dot{\epsilon}=0.1 \mathrm{~s}^{-1}$ the transient extensional response closely follows the linear viscoelastic envelope. The addi-

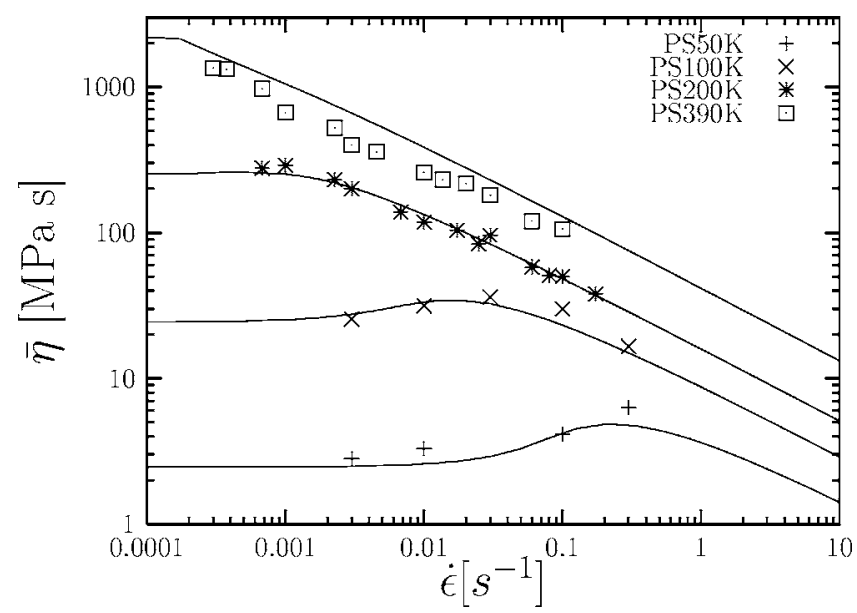

FIG. 10. Steady elongational viscosity as a function of the elongational rate for PS50K, PS100K, PS200K, and PS390K. All measurements performed at $130^{\circ} \mathrm{C}$. The solid lines are the predictions of the Wiest model. 


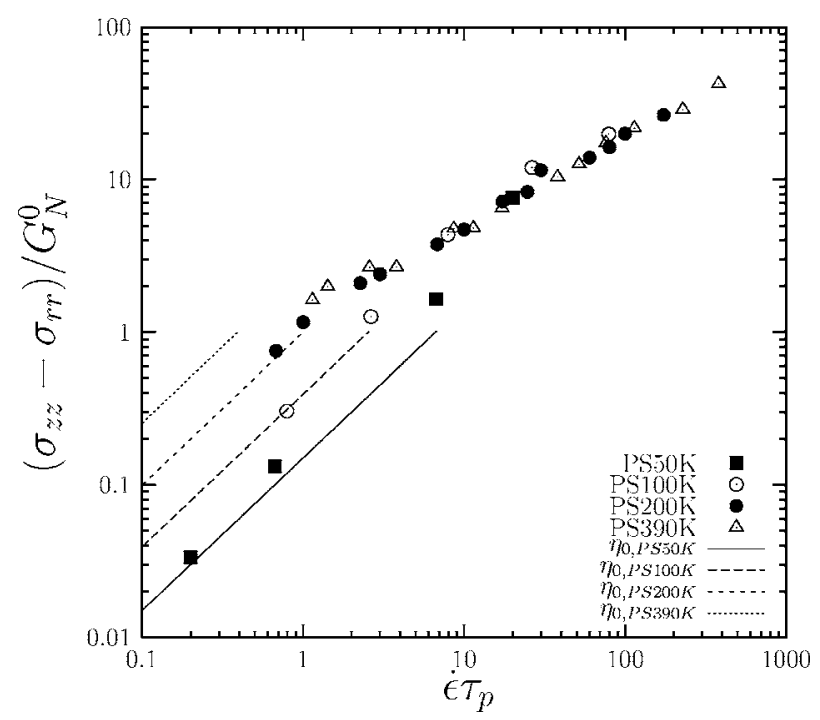

FIG. 11. The steady stress divided with the plateau modulus against the Marrucci-Deborah number $\epsilon \tau_{p}$ for PS50K, PS100K, PS200K, and PS390K.

tion of a small concentration of high molecular weight polymer to the blend (blend 1; $\left.\mathrm{c} / \mathrm{c}^{*}=2.5\right)$ results in a substantial transient strain hardening and also a steady extensional viscosity that is substantially above $3 \eta_{0}$ for the blend. That this additional stress is contributed by the higher molecular species can be easily demonstrated by examining the tensile stress contribution associated with a single mode upper convected Maxwell, (UCM) model (with modulus and relaxation time determined from Table III). This is shown by the dashed line in Fig. 12. As the concentration of higher molecular weight

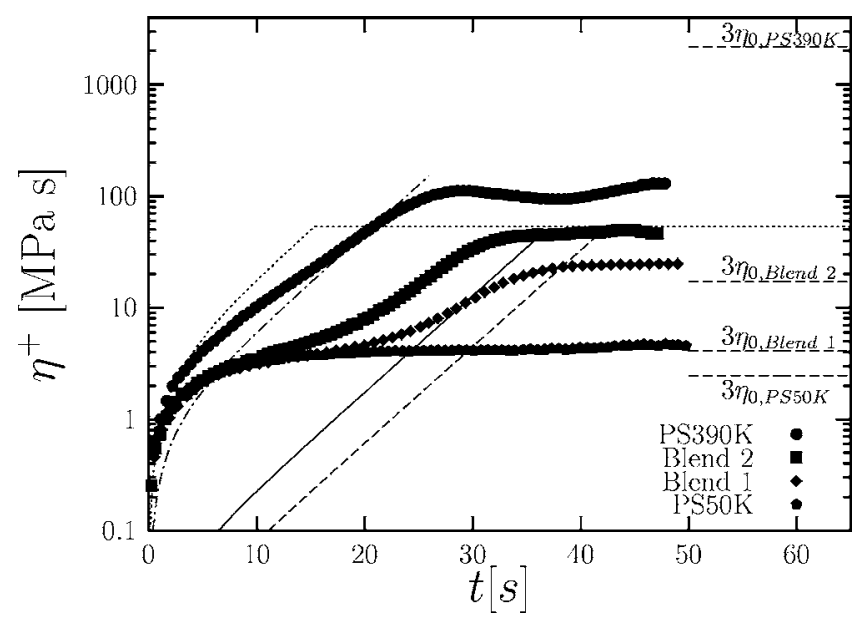

FIG. 12. Corrected transient extensional viscosities [equation (4)] of blend 1, blend 2, PS50K, and PS390K at $\dot{\boldsymbol{\epsilon}}=0.1 \mathrm{~s}^{-1}$. The broken line is the upper convected Maxwell (UCM) prediction for blend $1, \mathrm{De}=\lambda_{a, 2, \text { blend } 1}$ $\times 0.1 \mathrm{~s}^{-1}=121$, the solid line is the UCM prediction for blend $2 \mathrm{De}=\lambda_{a, 2 \text {,blend } 2} \times 0.1 \mathrm{~s}^{-1}=176$, the dashed dot line is the UCM prediction for PS390K De $=\lambda_{a, 1, \mathrm{PS} 390 \mathrm{~K}} \times 0.1 \mathrm{~s}^{-1}=1544$. The dotted line is the neo-Hookean model with $G=250 \mathrm{kPa}$, cut off at $\epsilon_{\max }$. The values of three times the zero shear viscosity for each melt is shown on the right with punctured lines. 


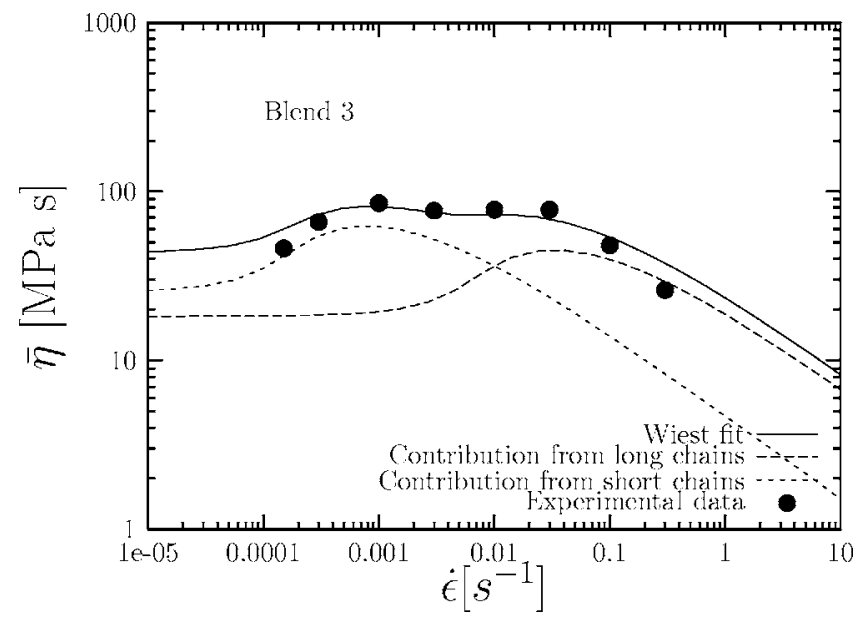

FIG. 13. Steady elongational viscosity against the elongational rate for blend 3. All measurements performed at $130{ }^{\circ} \mathrm{C}$. The solid line is the overall prediction of the Wiest model, and the dashed lines are the individual contributions from the two individual polymer species.

species is increased to $14 \%$ (blend 2) the magnitude of the extensional viscosity climbs further. Once again, we show the contribution of the high molecular weight species to the transient stress growth by plotting the response of an UCM model (solid line). The increase in the relaxation time of blend 2 also results in an increase in the Deborah number $\left(\mathrm{De}_{\mathrm{blend} 2}=1755 \mathrm{~s} \times 0.1 \mathrm{~s}^{-1}=176\right)$ and consequently the chains are fully elongated during the course of the experiment. This is illustrated by the horizontal dotted line in Fig. 12 which corresponds to cutting off the stress growth for blend 2 at a Hencky strain of $\epsilon_{\max }=\frac{1}{2} \ln \left(N_{K \text {,seg }}\right)=1.55$; see Appendix Sec. 2. Although the ultimate steady elongational viscosity shows some increase over three times the steady-state shear viscosity for this blend, the Trouton ratio is clearly reduced substantially compared to blend 1. Finally, we also show in Fig. 12 the transient response of the pure PS390K material at the same imposed stretch rate of $0.1 \mathrm{~s}^{-1}$, together with the UCM model (dashed-dot line). The material shows an initial linear viscoelastic response, followed by strain hardening but a steady elongational viscosity that is substantially less than $3 \eta_{0}$.

Plotting the steady elongational viscosity against elongational rate in Figs. 13-15, it is seen that the maximum in elongational viscosity is about $90 \%$ above $3 \eta_{0}$ for blend 3 , and about $700 \%$ above for blend 2 .

\section{CONSTITUTIVE MODELING OF THE STEADY ELONGATIONAL VISCOSITY}

The mathematical inconsistency mentioned in the Introduction can be resolved by acknowledging that the steady elongational viscosity for moderately entangled melts can have a maximum that exceeds $3 \eta_{0}$, the magnitude of the maximum depending on the molecular weight. The viscosity was found to scale with $\dot{\epsilon}^{-0.4}$ for large Deborah numbers, but where Bach et al. (2003a) claimed that this behavior starts at De $>1$, the results from PS100K show that this occurs at much higher Deborah numbers, De $>10$, and for PS50K even higher. It is thus clear that the shape of the steady elongational viscosity curve $\bar{\eta}$ is molecular weight dependent. The results from the blends show that the magnitude of the steady viscosity maximum becomes greater as the difference between the chain lengths in the blend increase. 


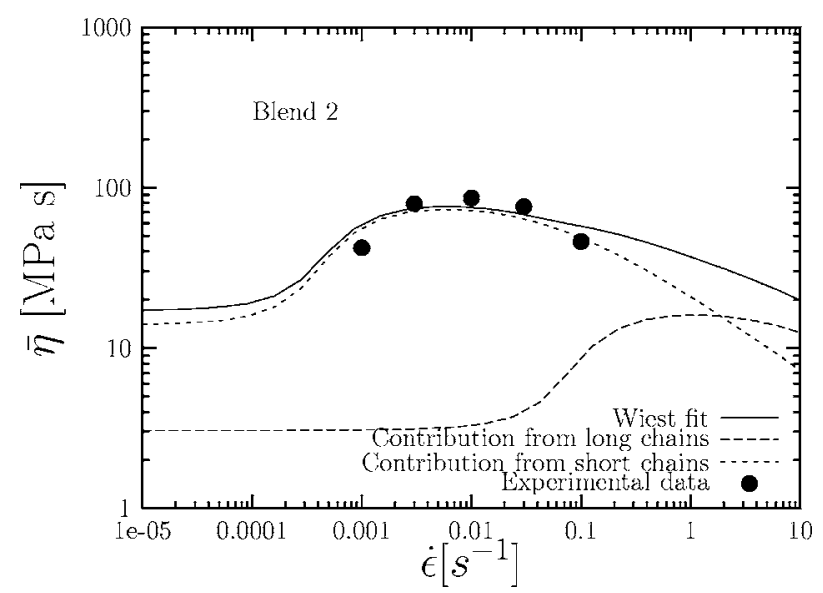

FIG. 14. Steady elongational viscosity against the elongational rate for blend 2. All measurements performed at $130{ }^{\circ} \mathrm{C}$. The solid line is the overall prediction of the Wiest model, and the dashed lines are the individual contributions from the two individual polymer species.

The behavior of the elongational viscosity for dilute solutions for high Deborah numbers has been studied by Gupta et al. (2000), who found that $\bar{\eta} \sim \dot{\epsilon}^{-0.5}$ for very diluted solutions of narrow molar mass distribution polystyrene. This result can be modeled theoretically by including finite extensibility into the Giesekus (1982) anisotropic friction dumbbell model to account in an average fashion for the orientation of the surrounding molecules (Wiest, 1989). The asymptotic analysis is performed in detail in the Appendix. Marrucci and Ianniruberto (2004) quantitatively predict the asymptotic stress behavior observed experimentally by incorporating chain squeeze into their model. This will essentially give rise to anisotropic friction too, and the Wiest model is a simple way of describing this.

The constitutive model in terms of integral average of the connector dyad $\langle\mathbf{Q Q}\rangle$ is

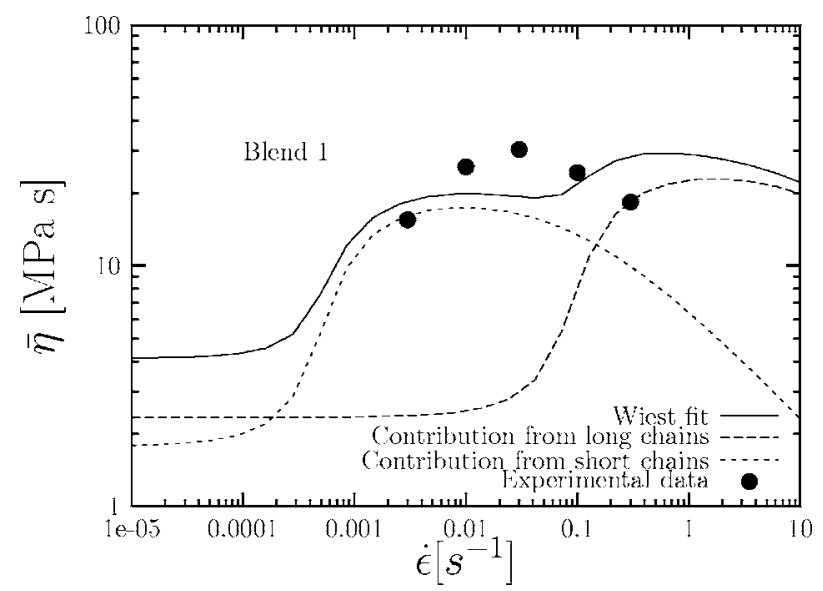

FIG. 15. Steady elongational viscosity against the elongational rate for blend 1 . All measurements performed at $130{ }^{\circ} \mathrm{C}$. The solid line is the overall prediction of the Wiest model, and the dashed lines are the individual contributions from the two polymers. 


$$
\begin{aligned}
\langle\mathbf{Q Q}\rangle_{(1)} & =-\frac{4 H}{\zeta^{-1}}\left(f\langle\mathbf{Q Q}\rangle-\frac{k T}{H} \boldsymbol{I}\right) \\
& =4 k T \zeta^{-1}-4 H\langle\mathbf{Q Q}\rangle f \boldsymbol{\zeta}^{-1},
\end{aligned}
$$

where the Giesekus mobility tensor is

$$
\boldsymbol{\zeta}^{-1}=\frac{1}{\zeta}\left(\boldsymbol{\delta}-\frac{a}{n k T} \boldsymbol{\tau}_{p}\right)
$$

and $f$ describes the nonlinearity of the Warner spring in the FENE-P dumbbell model,

$$
f=\left[1-\frac{\left\langle Q^{2}\right\rangle}{Q_{0}^{2}}\right]^{-1}
$$

where $\left\langle Q^{2}\right\rangle=\operatorname{tr}\langle\mathbf{Q Q}\rangle$. Here, $H$ is a spring constant, $n$ is the number density of dumbbells, $k$ is Boltzmann's constant, $T$ the absolute temperature, $\boldsymbol{I}$ is the unit tensor, and $Q_{0}$ is the maximum length of the dumbbell. The stress tensor for the polymer is given by Eq. (13.7-5) of Bird et al. (1987),

$$
\tau_{p}=-n H f\langle\mathbf{Q Q}\rangle+n k T \boldsymbol{\delta} .
$$

By elimination of $\langle\mathbf{Q Q}\rangle$ a constitutive equation in terms of the polymeric stress, $\boldsymbol{\tau}_{p}$, may be obtained in the form

$$
\left(Z-\lambda_{H} \frac{D \ln Z}{D t}\right) \boldsymbol{\tau}_{p}+\lambda_{H} \boldsymbol{\tau}_{p,(1)}-\frac{a Z}{n k T}\left(\boldsymbol{\tau}_{p} \cdot \boldsymbol{\tau}_{p}\right)=-n k T \lambda_{H}\left(\dot{\gamma}+\frac{D \ln Z}{D t} \boldsymbol{\delta}\right),
$$

where

$$
Z=\frac{1}{b}\left(b+3-\frac{\operatorname{tr} \tau_{p}}{n k T}\right),
$$

$\dot{\gamma}$ is the strain rate tensor, and $b$ is the finite extensibility parameter for the entanglement segment given by $b=H_{\text {seg }} Q_{0}^{2} /(k T)$. The single time constant of the model is $\lambda_{H}=\zeta /\left(4 H_{\mathrm{seg}}\right)$. The zero shear viscosity is found (Wiest, 1989) to be $\eta_{0}=n k T \lambda_{H} b /(b$ $+3)$.

This model has three free parameters, $a, b$, and $\lambda_{H}$, where $a$ is a dimensionless scalar between 0 and 1 describing the degree of anisotropy in the hydrodynamic drag in the melt; when $a=0$ the drag is completely isotropic while $a=1$ corresponds to maximum anisotropy. The model describes the dynamics of one entangled segment. The finite extensibility parameter $b$ is expected to be independent of molecular weight, see the Appendix, Sec. 2 and equal to three times the number of Kuhn steps in a entanglement segment, $N_{k, \text { seg }}$, and $\lambda_{H}$ is a characteristic time constant. By solving the constitutive equation for uniaxial elongational flow, one sees that by changing the $a$ parameter from 0 to 1 at fixed values of $b$ and $\lambda_{H}$, the steady elongational viscosity $\bar{\eta}$ has a maximum above $3 \eta_{0}$, whose magnitude increases as $a \rightarrow 0$, and decreases and almost disappears as $a \rightarrow 1$.

Relating the maximum in $\bar{\eta}$ with drag anisotropy for monodisperse melts may help rationalize why the local maximum is almost absent for high molecular weight melts, such as PS390K, and becomes increasingly large with lower molecular weights. If the magnitude of $a$ is interpreted as a potential for anisotropy, one would intuitively assume that for a $100 \%$ stretched and aligned polymer melt, which would be the case at steady 
state for infinite elongational rate, the anisotropy inside the melt would be largest in the limit of long chains. A melt of shorter, but still stretched and aligned chains, would have a higher density of free ends, thereby reducing anisotropy.

The same arguments can be used for bidisperse melts. Blends 1 and 2 contain the same polymers, but the long chains are more diluted by short chains in blend 1 and we would expect the $a$ parameter for blend 2 to be larger than for blend 1, since the potential for anisotropic drag is lowest when the longer chains are surrounded by fewer long chains. Blends 2 and 3 have the same mass fraction of PS390K, but are mixed with PS50K and PS100K chains, respectively. Again, we expect the anisotropic parameter $a$ to be smallest for blend 2, which is the case as shown later. This effect is more pronounced compared to the difference between blends 1 and 2 .

The question is now whether or not the model is able to explain the data quantitatively. If the model is fitted to results of the monodisperse melts, ideally only two parameters should be fitted, $a$ and $\lambda_{H}$, since $b$ is related to the number of Kuhn steps in an entanglement segment which is known. It is not expected that a single mode version of the model will describe the complete transient elongational viscosity because the initial transient growth in the stress is related to the LVE behavior, and the Wiest model is essentially a single time constant model with inclusion of anisotropy and a FENE-P spring between the dumbbells. A multimode version would be needed to quantitatively describe the LVE behavior. Since we are concerned primarily with the steady elongational viscosity, only one mode is used in this analysis.

PS100K is the melt with the most elongational measurements above and below $\dot{\epsilon}=1 / \tau_{d}$, i.e., at intermediate Deborah numbers, which in this work is the most interesting area. To obtain an idea of the relative magnitude of the different constitutive parameters, a fit to the elongational viscosity data for PS100K is made by changing $a, b$, and $\lambda_{H}$, and a separate fit where $b$ is kept constant at $3 N_{k \text {,seg }}$ and only $a$ and $\lambda_{H}$ are allowed to change. Bach et al. (2003a) reported the number of Kuhn steps between entanglement segments as $N_{k, \text { seg }}=22$, which makes $b=66$. The result is shown in Fig. 16, and the fitted values are shown in the caption. Both fits give reasonable agreement with the experimental data. The time constant $\lambda_{H}$ is in both fits of about the order of the expected reptation time in both fits, around $100 \mathrm{~s}$, and $a$ is in the expected interval between 0 and 1 . In contrast to the limiting case of the Giesekus model $(b \rightarrow \infty)$, the Wiest model does not predict an unphysical degree of shear thinning in the steady shear viscosity for $a>0.5$, (Wiest, 1989). Instead, it is found that the shear stress plateaus at high shear rates, corresponding to the steady shear viscosity decreasing as $\dot{\gamma}^{-1}$.

The least-square fitted value of $b$ corresponds to very little extensibility, which appears unphysical. Since we find no consistency in the magnitude of $b$, this parameter is allowed to float in the following fits of the experimental data to the model.

The value of $\lambda_{H}$ does seem to resemble the reptation time, and in the following fits the value of $\lambda_{H}$ is held fixed at the pre-determined value of $\lambda_{a}$. The experimental data do, as mentioned before, show that the steady elongational viscosity for high Deborah numbers scales with $M$ between PS390K and PS200K, and that the steady elongational viscosity $\bar{\eta} \sim \dot{\epsilon}^{-0.4}$. If it is assumed that the former scaling also applies for $\bar{\eta}$ between the PS200K and PS100K, this can be used as a constraint fitting the steady viscosities for PS100K to the Wiest model. This is essentially the same as weighting the two largest elongational rate measurements most heavily, since these do confirm the experimentally determined molecular weight scaling between PS200K and PS100K, and also exhibit a decrease as $\bar{\eta} \sim \dot{\epsilon}^{-0.4}$. The fitted parameters of the Wiest model, with the above-mentioned constraint regarding molecular weight scaling, together with the choices of $\lambda_{a}$ are given in Table IV 


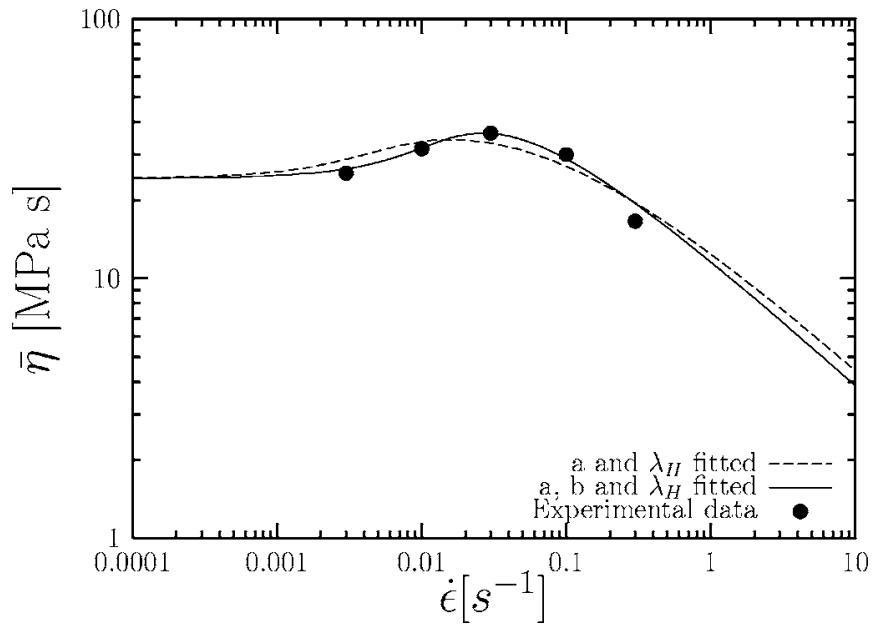

FIG. 16. Steady extensional viscosity measurements of PS100K $(\bullet)$ measured at $130{ }^{\circ} \mathrm{C}$. The solid line is the Wiest fit where $a=0.1805, b=4.44$ and $\lambda_{H}=66.85 \mathrm{~s}$. The dotted line is the Wiest fit where $a=0.4055, b=66$ and $\lambda_{H}=105.7725 \mathrm{~s}$.

(see also Fig. 10). The blend data for blends 1, 2, and 3 are least-square fitted to the Wiest model by using two time constants and two zero shear viscosities, but no constraints on the molecular weight scaling as seen in Figs.13-15.

From the data shown in Table IV for the monodisperse melts, it is seen that the value of $a$ is unity for PS390K and gradually decreases as the molecular weight goes down, ending at $a=0.14$ for PS50K, indicating that the degree of molecular anisotropy in the drag force falls as molecular weight goes down, which was expected due to the higher density of dangling ends. The magnitudes of the fitted values of $b$ for the monodisperse melts do not show any general trend with $M_{w}$, confirming that this parameter is not correlated with the molecular weight. We have also included in the table the values of the maximum in the steady elongational viscosity relative to $3 \eta_{0}$, and the maximum in $\bar{\eta}_{\max } /\left(3 \eta_{0}\right)$ appears to vary inversely with $a$. The strain hardening behavior we see for the low molecular weight melts and the bidisperse blends is therefore related in the terms of the Wiest model to the degree of anisotropy in the elongated melt.

The results for the blends show that it is possible to fit the data successfully to a two-mode Wiest model to the blend 3 melt but not to the blend 1 melt. If a single-mode fit is used instead, with all the parameters varying, a much better fit is obtained.

TABLE IV. The least-square fit of the Wiest model parameters $a$ and $b$ for the different melts together with dimensionless maximum in the steady elongational viscosity $\bar{\eta}_{\max } /\left(3 \eta_{0}\right)$.

\begin{tabular}{lllll|ccc}
\hline \multicolumn{1}{c}{ Name } & PS50K & PS100K & PS200K & PS390K & Blend 1 & Blend 2 & Blend 3 \\
\hline w/w\% PS50K & & & & & 95.98 & 85.63 & 0 \\
w/w\% PS100K & & & & & 0 & 0 & 85.98 \\
w/w\% PS390K & & & & & 4.02 & 14.37 & 14.02 \\
$a$ & 0.1372 & 0.2182 & 0.7033 & 1.000 & $1.89 \times 10^{-4}$ & $5.65 \times 10^{-4}$ & 0.1982 \\
$b$ & 9.9 & 4.6 & 5.3 & 6.93 & 13.3 & 5.9 & 98.5 \\
$\bar{\eta}_{\max } /\left(3 \eta_{0}\right)$ & 2.56 & 1.54 & 1.17 & 1 & 7.40 & 5.06 & 1.95 \\
\hline \hline
\end{tabular}




\section{CONCLUSION}

The steady elongational viscosity of two moderately entangled monodisperse polystyrene melts, with molecular weights of 52 and $103 \mathrm{~kg} /$ mole, have been measured for elongational deformation rates ranging from $\dot{\epsilon}=0.003$ to $\dot{\epsilon}=0.3 \mathrm{~s}^{-1}$. It is observed that the steady elongational viscosity vs elongational rate goes through a maximum, and is followed by a decreasing region in which the elongational rate scales as $\bar{\eta} \sim M_{w} \dot{\epsilon}^{-0.4}$ for large elongational rates. The maximum is the result of fewer entanglements in these melts, in agreement with the predictions of Marrucci and Ianniruberto (2004).

The steady elongational viscosity has also been measured for bidisperse blends of a high and a low molecular weight monodisperse polystyrene. Here, we also observe a maximum in the steady elongational viscosity vs elongational rate. This maximum, relative to three times the zero shear viscosity, increases as the concentration of high molecular weight chains decreases. This observation is contrary to that reported by Wagner et al. (2005), who found that the strain hardening increased with increasing concentration of ultrahigh molecular weight polystyrene. The molar masses in their studies are, however, well above $365 \mathrm{~kg} /$ mole which may be argued to be the upper limit for the application of the Wiest model; see the Appendix, Sec. 3. Conversely, the maximum in steady elongational viscosity against elongational rate increases with reduced molecular weight of the low molecular weight chains.

The maximum found for our bidisperse polymer blends indicates a qualitative difference between monodisperse and bidisperse melts. This is different from the corresponding situation between monodisperse and bidisperse solutions (Ye et al., 2003) with Graessley parameters $G_{r} \ll G_{r c}$.

The fact that the steady elongational viscosity of a blend of long (390 kg/mole) and short polystyrene chains exhibits a maximum as function of elongation rate, while the melt of pure long chains does not, may be interpreted in terms of the Wiest dumbbell model which combines the Giesekus anisotropic friction concept with finite extensibility. Indeed, the pure melt of long chains has a large potential for anisotropic drag corresponding to a Giesekus parameter $a=1$. Conversely, in blends with a significantly lower molar mass or even entangled solutions, the long chains will encounter an environment with less potential for anisotropy. Basically the long chains undergo stretching at extensional rates that are small enough that the shorter chains are not oriented, thereby providing an isotropic drag environment.

\section{ACKNOWLEDGMENTS}

The authors gratefully acknowledge financial support to the Graduate School of Polymer Science from Danish Research Training Council and the Danish Technical Research Council to the Danish Polymer Centre.

\section{APPENDIX}

\section{Behavior of the Wiest model for $\dot{\epsilon} \rightarrow \infty$}

The constitutive equation for homogeneous steady flow of the Wiest model is

$$
Z \tau_{p}+\lambda_{H} \tau_{1}-\frac{a Z}{n k T}\left(\tau_{p} \cdot \tau_{p}\right)=-n k T \lambda_{H} \dot{\gamma}
$$


In strong uniaxial elongation steady flows, the only stress contribution to the forces in the melt is $\tau_{z z}$. To solve for $\tau_{p, z z}$ the variable substitution $y=-\tau_{p, z z} /(n k T)$, and $x=\lambda_{H} \dot{\epsilon}$ is introduced. The stress in the $z z$ direction then becomes

$$
-\frac{a y^{3}}{b}+\frac{y^{2}}{b}+2 x y+2 x=0 .
$$

Since $\tau_{z z}$ in stretching is negative, $y>0$ for all values of $x$. It is assumed that, for large elongational rates, the viscosity, and thereby also $y$ behaves as a power law-function i.e., $y \sim A x^{\alpha}$ for $x \rightarrow \infty$. Substituting this into Eq. (A2) we get

$$
-\frac{a A^{3} x^{3 \alpha}}{b}+\frac{A^{2} x^{2 \alpha}}{b}+2 A x^{1+\alpha}+2 x=0 .
$$

Since the absolute value of the stress, $\left|\tau_{z z}\right|$, and therefore $y$, increases for increasing elongational rates, $\alpha$ must be larger than zero. The largest terms in Eq. (A3) are $2 A x^{1+\alpha}$ and $-a A^{3} x^{3 \alpha} / b$ which have to balance as $x \rightarrow \infty$, whereby we obtain $\alpha=1 / 2$.

The pre-exponential terms also have to balance, for $x \rightarrow \infty$ so the parameter $A$ becomes

$$
2 A=\frac{a}{b} A^{3} \Rightarrow A=\sqrt{\frac{2 b}{a}} .
$$

The final asymptotic result is that

$$
\frac{\bar{\eta}}{n k T \lambda_{H}}=\sqrt{\frac{2 b}{a}}\left(\lambda_{H} \dot{\epsilon}\right)^{-1 / 2} \text { for } \quad \dot{\epsilon} \rightarrow \infty .
$$

The modified Giesekus model thereby gives a physical explanation for the fact that $\bar{\eta} \sim \dot{\epsilon}^{-1 / 2}$ for high elongational rates, whereas the simple Giesekus model predicted $\bar{\eta}$ as having a finite limit for infinite $\dot{\epsilon}$.

\section{Molecular interpretation of the finite extensibility $b$-parameter}

We apply the Wiest model to a representative single entangled tube segment of the melt. The chain in the tube segment is modeled as a FENE spring with maximum length $Q_{0}$ and spring constant $H_{\text {seg }}$ given by

$$
Q_{0}=N_{k, \mathrm{seg}} L_{k},
$$

and

$$
H_{\mathrm{seg}}=\frac{3 k T}{N_{k, \mathrm{seg}} L_{k}^{2}},
$$

where $N_{k \text {,seg }}$ is the number of Kuhn steps in an entanglement segment, and $L_{k}$ is the length of each Kuhn step. From these equations the constant $b$ is defined, which yields a simpler expression,

$$
b \equiv \frac{H_{\mathrm{seg}} Q_{0}^{2}}{k T}=3 N_{k, \mathrm{seg}}
$$

The finite strain extensibility of an entanglement segment is given by $\exp \left(\epsilon_{\max }\right)$ $=\sqrt{N_{k \text {,seg }}}$, Fang et al. (2000), which means that in the affine limit, that is at infinite elongational rate, the segment has reached its maximum stretch at a strain of 


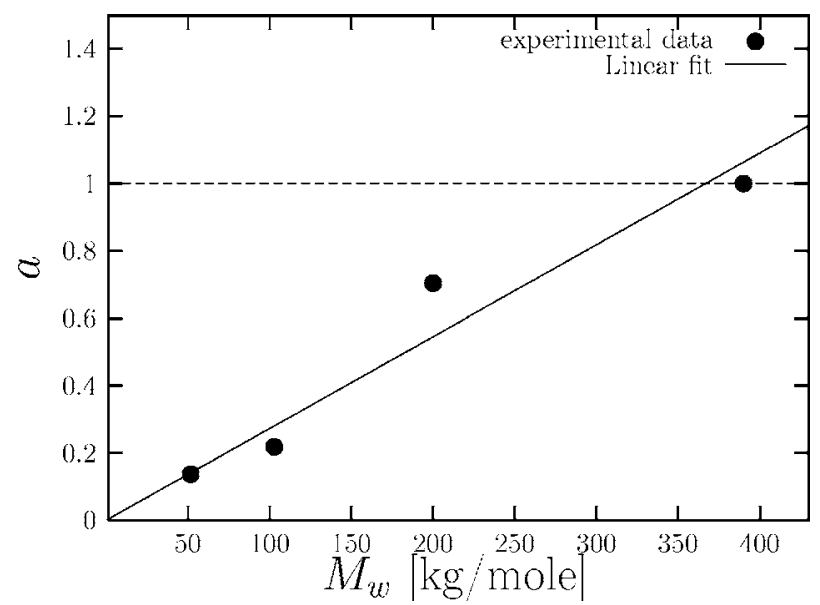

FIG. 17. Least-square fitted parameter of $a$ against $M_{w}$. Solid line is a linear fit, $a=A\left(M_{w}\right)^{1}$.

$$
\epsilon_{\max }=\frac{1}{2} \ln N_{k, \mathrm{seg}}=\frac{1}{2} \ln \left(\frac{1}{3} b\right)
$$

\section{Scaling of steady state stress with $M_{w}$ in the Wiest model}

For an entangled polymer melt, the prefactor scale for stress is independent of molecular weight: $n k T \equiv G_{N}^{0}=\rho R T / M_{e}$. With respect to the time constant $\lambda_{H}$, the relevant times to consider would be either the reptation time, which is the characteristic time of the entire chain in the constrained tube taken from the Doi-Edwards interpretation of a polymer melt, or the Rouse time, which is a time constant for the stretching of the entangled chain. It makes sense in the Wiest model to choose a Rouse scaling for $\lambda_{H}$ since it describes stretching which would make $\lambda_{H} \sim M_{w}^{2}$. However, fitting showed that $\lambda_{H} \simeq \lambda_{a}$, which suggests that $\lambda_{H}$ scales as the Doi-Edwards reptation time, i.e., $\lambda_{H}$ $\sim M_{w}^{3}$. Of course, such apparent inconsistencies are inevitable with a dumbbell-based segment-level model. More detailed constitutive models for monodisperse melts (Marrucci and Ianniruberto, 2004) recognize that the time scales for orientation and chain stretching scale differently with molecular weight. This is beyond the scope of the present discussion. We seek simply to show that a simple model with anisotropic drag such as the Wiest model is capable of describing the experimental observations in pure melts and in blends. As we have noted above, the extensibility $b$ is only a function of the length of an entangled segment and is therefore independent of $M_{w}$.

The steady stress scaling then becomes:

$$
\begin{aligned}
& \left(\sigma_{z z}-\sigma_{x x}\right)=\bar{\eta} \dot{\boldsymbol{\epsilon}}=G_{N}^{0} \quad \cdot \quad \lambda_{H}^{1 / 2} \quad \cdot \quad b^{1 / 2} \quad \cdot a^{-1 / 2} \dot{\boldsymbol{\epsilon}}^{1 / 2} \sqrt{2} \\
& \sim\left(M_{w}^{0}\right) \cdot\left(M_{w}^{3}\right)^{1 / 2} \cdot\left(M_{w}^{0}\right)^{1 / 2} \cdot\left(M_{w}^{x}\right)^{-1 / 2}
\end{aligned}
$$

Assuming that the tensile stress difference scales as $\left(\sigma_{z z}-\sigma_{x x}\right) \sim M_{w} \dot{\epsilon}^{1 / 2}$, an expression for molecular weight scaling-factor $x$ of $a$ is found using Eq. (A10) to be $a \sim M_{w}^{1}$. This is only valid as long as $a \leq 1$.

Figure 17 below shows the fitted values of $a$ as function of the molecular weight $M_{w}$. The solid line is the best linear fit against molecular weight, i.e., $a=A M_{w}^{1}$. 
It is not possible to validate the molecular weight scaling of $a$ from the plot, since only three data points are available. But, the plot does indicate that the Wiest model cannot be applied as a constitutive equation for polystyrenes with molecular weights more than around $365 \mathrm{~kg} / \mathrm{mole}$ and this also explains why the fit for the steady elongational viscosity for PS390K was so poor. The drag anisotropy appears to saturate for entangled polystyrene melts with molecular weights above $365 \mathrm{~kg} / \mathrm{mole}$.

\section{References}

Bach, A., H. K. Rasmussen, and O. Hassager, "Extensional viscosity for polymer melts measured in the filament stretching rheometer," J. Rheol. 47, 429-441 (2003a).

Bach, A., K. Almdal, H. K. Rasmussen, and O. Hassager, "Elongational viscosity of narrow molar mass distribution polystyrene," Macromolecules 36, 5174-5179 (2003b).

Baumgaertel, M., A. Schausberger, and H. H. Winter, "The relaxation of polymers with linear flexible chains of uniform length," Rheol. Acta 29, 400-408 (1990).

Bird, R. B., C. F. Curtiss, R. C. Armstrong, and O. Hassager, Dynamics of Polymeric Liquids, Vol. 2 Kinetic Theory (Wiley, New York, 1987).

des Cloizeaux, J., “Double reptation vs. simple reptation in polymer melts," Europhys. Lett. 5, 437-442 (1988).

Doi, M., Introduction to Polymer Physics (Clarendon, Oxford, 1992).

Doi, M., and S. F. Edwards, The Theory of Polymer Dynamics (Clarendon, Oxford, 1986).

Doi, M., W. W. Graessley, E. Helfand, and D. S. Pearson, "Dynamics of polymers in polydisperse blends," Macromolecules 20, 1900-1906 (1987).

Eriksson, T., and H. K. Rasmussen, "The effects of polymer melt rheology on the replication of surface microstructures in isothermal moulding," J. Non-Newtonian Fluid Mech. 127, 191-200 (2005).

Fang, J., M. Kröger, and H. C. Öttinger, "A thermodynamically admissible reptation model for fast flows of entangled polymers. II. Model predictions for shear and extensional flows," J. Rheol. 44, 1293-1317 (2000).

Fetters, L. J., D. J. Lohse, D. Richter, T. A. Witten, and A. Zirkel, "Connection between polymer molecular weight, density, chain dimensions, and melt viscoelastic properties," Macromolecules 27, 4639-4647 (1994).

Giesekus, H., "A simple constitutive equation for polymer fluids based on the concept of deformationdependent tensorial mobility," J. Non-Newtonian Fluid Mech. 11, 69-109, (1982).

Gupta, R. K., D. A. Nguyen, and T. Sridhar, "Extensional viscosity of dilute polystyrene solution—effect of concentration and molecular weight," Phys. Fluids 12, 1296-1318 (2000).

Ianniruberto, G., and G. Marrucci, "A simple constitutive equation for entangled polymers with chain stretch," J. Rheol. 45, 1305-1318 (2001).

Jackson, J. K., and H. H. Winter, "Entanglement and flow behavior of bidisperse blends of polystyrene and polybutadiene," Macromolecules 28, 3146-3155 (1995).

Kolte, M. I., H. K. Rasmussen, and O. Hassager, "Transient filament stretching rheometer II: Numerical simulation," Rheol. Acta 36, 285-302 (1997).

Lee, J. H., L. J. Fetters, L. A. Archer, and A. F. Halasa, "Tube dynamics in binary polymer blends," Macromolecules 38, 3917-3932, (2005).

Likhtman, A., "Single-chain slip-link model of entangled polymers: simultaneous description of neutron spinecho, rheology, and diffusion," Macromolecules 38, 6128-6139 (2005).

Luap, C., C. Müller, T. Schweizer, and D. C. Venerus, "Simultaneous stress and birefringence measurements during uniaxial elongation of polystyrene melts with narrow molecular weight distribution," Rheol. Acta 45, 83-91 (2005).

Marrucci, G., and N. Grizzuti, "Fast flows of concentrated polymers-Predictions of the tube model on chain stretching," Gazz. Chim. Ital. 118, 179-185 (1988).

Marrucci, G., and G. Ianniruberto, "Interchain pressure effect in extensional flows of entangled polymer melts," 
Macromolecules 37, 3934-3942 (2004).

Mead, D. W., R. G. Larson, and M. Doi, "A molecular theory for fast flows of entangled polymers," "Macromolecules 31, 7895-7914 (1998).

Milner, S. T., and T. C. B. McLeish, "Reptation and contour-length fluctuations in melts of linear polymers," Phys. Rev. Lett. 81, 725-728 (1998).

Ndoni, S., C. M. Papadakis, F. S. Bates, and K. Almdal, "Laboratory-scale setup for anionic polymerization under inert atmosphere," Rev. Sci. Instrum. 66, 1090-1095, (1995).

Park, S. J., and R. G. Larson, "Tube dilation and reptation in binary blends of monodisperse linear polymers," Macromolecules 37, 597-604 (2004).

Rasmussen, H. K., and O. Hassager, "The role of surface tension on the elastic decohesion of polymeric filaments, J. Rheol. 45, 527-537 (2001).

Rasmussen, H. K., J. K. Nielsen, A. Bach, and O. Hassager, "Viscosity overshoot in the start-up of uni-axial elongation of LDPE melts,” J. Rheol. 49, 369-381 (2005).

Rasmussen, H. K., J. H. Christensen, and S. J. Gottsche, "Inflation of polymer melts into elliptic and circular cylinders," J. Non-Newtonian Fluid Mech. 93, 245-263 (2000).

Schausberger, A., and G. Schindlauer, "Linear elastico-viscous properties of molten standard polystyrenes," J. Rheol. 24, 220-227 (1985).

Schieber, J., J. Neergaard, and S. Gupta, "A full-chain, temporary network model with sliplinks, chain-length fluctuations, chain connectivity and chain stretching," J. Rheol. 47, 213-233 (2003).

Spiegelberg, S. H., and G. H. McKinley, "The role of end-effects on measurements of extensional viscosity in filament stretching rheometers," J. Non-Newtonian Fluid Mech. 64, 229-267 (1996).

Struglinski, M. J., and W. W. Graessley, "Effects of polydispersity on the linear viscoelastic properties of entangled polymers. I. Experimental observations for binary mixtures of linear polybutadiene," Macromolecules 18, 2630-2643 (1985).

Szabo, P., "Transient filament stretching rheometer. I: force balance analysis,” Rheol. Acta 36, 277-284 (1997).

Szabo, P., and G. H. McKinley, "Filament stretching rheometer: Inertia compensation revisited," Rheol. Acta 42, 269-272 (2003).

Wagner, M. H., S. Kheirandish, K. Koyama, A. Nishioka, A. Minegishi, and T. Takahashi, "Modeling strain hardening of polydisperse polystyrene melts by molecular stress function theory," Rheol. Acta 44, 235-243 (2005).

Wagner, M. H., S. Kheirandish, and O. Hassager, "Quantitative prediction of the transient and steady-state elongational viscosity of nearly monodisperse polystyrene melts,” J. Rheol. 49, 1317-1327 (2005).

Wiest, J. M., "A differential constitutive equation for polymer melts, Rheol. Acta 28, 4-12 (1989).

Ye, X., R. G. Larson, C. Pattamaprom, and T. Sridhar, "Extensional properties of monodisperse and bidisperse polystyrene solutions," J. Rheol. 47, 443-468 (2003). 